\title{
Desktop flight simulators: Simulation fidelity and pilot performance
}

\author{
DRAKE R. BRADLEY and STUART B. ABELSON \\ Bates College, Lewiston, Maine
}

\begin{abstract}
Improvements in the computing power and visual resolution of modern desktop computing systems, as well as advances in software technology for displaying high-speed animations, have encouraged the development of relatively sophisticated real-time flight simulators for the PC and Macintosh. We review some of the factors that determine how well such programs capture the actual experience of flight. The most significant factor limiting the quality of performance in flying a simulated aircraft is the "frame rate" problem: at low altitudes and in highly detailed visual environments, as in approaching a runway threshold during landing, the computational demands of the animation may necessitate a reduction in the number of frames displayed per second on the screen. The delayed sensory feedback that results proves to be very detrimental to sustaining smooth control of the aircraft, especially during the flare to touchdown where such control is needed most. This finding parallels the well-known effects of delayed auditory feedback (Lee, 1950) and delayed visual feedback (Smith, 1962).
\end{abstract}

For many aviation enthusiasts, the challenge and excitement of learning to fly is tempered only by the cost of instruction ( $\$ 60-\$ 80 /$ hour). However, recent advances in hardware and software technology make it possible to run highly sophisticated flight simulator programs on desktop computers. A flight simulator is a computer program that models all of the important principles of operating an aircraft in three-dimensional flight. The typical simulator allows the user to take off, fly the aircraft through a variety of flight configurations (including aerobatics), navigate between way points, and land at a destination. Some programs allow the user to control the seasonal, weather, and wind conditions of the flight, the reliability of the aircraft (so that simulated "emergencies" can arise), and various other flight parameters. While some simulators are intended only for entertainment, others are specifically designed for flight training. Many provide real-world navigation capability using databases and scenery areas that cover the United States, Canada, and Western Europe. Major terrain features, such as lakes, rivers, highways, and cities, as well as airports and navaids, are provided in the databases.

Most desktop flight simulators are reasonably good at modeling the flight dynamics and "feel" of general avia-

We thank American Airlines (Dallas), and Lt. Gleysteen, Lt. Stucky, and Gary Hensley of Brunswick Naval Airstation (Brunswick, ME) for providing the authors access to full motion flight simulators (an MD80 and Orion P-3, respectively). The time spent exploring these simulators helped the authors to better appreciate the unique contributions and limitations of desktop flight simulators. We also thank Thomas Kopke for reviewing this paper and providing comments and suggestions on technical matters. Any errors that remain are the responsibility of the authors. Correspondence should be addressed to D. R. Bradley, Department of Psychology, Bates College, Lewiston, ME 04240 (e-mail: dbradley@abacus.bates.edu). tion aircraft, at depicting the behavior of the various instruments in the cockpit, and at displaying a real-time animation of the view out the window. Not surprisingly, desktop simulators focus on modeling the visual experience of flight, since this can be depicted on the computer monitor. Due to hardware and cost constraints, auditory cues are less frequently modeled, and sensory cues affecting the vestibular and kinesthetic systems are not modeled at all. The realism of the visual display continues to improve, however, and the industry has come a very long way from initial efforts, which used "wireframe" drawings of buildings and other objects. Recent programs such as Microsoft Flight Simulator 5.0 use photorealistic imaging, cybergraphics, real-time ray tracing, Gouraud shading, fractal geometry, and other advanced graphics techniques (Mass, 1993; Pruyn, 1992). The result is stunningly realistic images and real-time animation performance that rivals that previously seen only on dedicated mainframe systems. In light of these advances, the authors became intrigued with the possibility of using desktop flight simulators to promote skill acquisition and positive transfer to the actual flight environment. Moreover, we see intriguing possibilities for conducting research investigating the acquisition of complex cognitive skills in an ecologically valid setting (i.e., learning to fly and navigate an aircraft). The present paper reviews the strengths and limitations of desktop flight simulators from this perspective.

\section{How Simulators Work}

The reader unfamiliar with flight simulators might wonder how the airplane is flown using a computer. The pilot employs a mouse, control yoke, joystick, or cursor keys to control the pitch and bank of the aircraft, and various keys on the keyboard to adjust the throttle, gear, 
flaps, radios, and so on. The program monitors the inputs from the flight controls, computes their effects on the aircraft's attitude, airspeed, altitude, and the like, and then displays these effects on the instruments and in the view out the window. This cycle is repeated many times each second, so what we see on the computer monitor is a succession of snapshots or frames of what the view inside and outside of the cockpit looks like at discrete points in time. At a typical rate of 15-30 frames per second (fps), an impression of continuous movement of the aircraft through space is achieved. The apparent motion is simply a variant of the phi phenomenon (Wertheimer, 1912/1961).

In the real world, flying by reference to visual cues outside the cockpit is conducted under visual flight rules (VFR), whereas flying solely by reference to the instruments is conducted under instrument flight rules (IFR). There are programs that simulate VFR flight (e.g., Electronic Arts' Advanced Flight Trainer), IFR flight (e.g., AzureSoft's ELITE flight simulator), or both (e.g., Microsoft's Flight Simulator 5.0). The most sophisticated and expensive simulators are those designed for IFR flight: AzureSoft's ELITE flight simulator, for example, is sufficiently advanced that the FAA is considering the possibility of allowing this simulator to be used for flight training (Forster, 1991).

Depending on the sophistication of the simulator, several techniques may be available for navigating the aircraft. The simplest method, called pilotage, consists of comparing visual landmarks seen "out of the cockpit" to corresponding features on aeronautical charts or maps. With dead reckoning, the pilot navigates by flying specific headings on a compass, and by using time and distance calculations to estimate the time between various check points. Finally, radio navigation allows the pilot to navigate using electronic aids, such as VOR (very high frequency omnidirectional range), ADF (automatic direction finding), LORAN (long-range navigation), GPS (global positioning systems), and ILS (instrument landing systems). Both dead reckoning and radio navigation permit the pilot to navigate from point to point on instruments (i.e., without reference to visual landmarks on the ground). Hence, these navigation techniques must be employed for IFR flight. In contrast, VFR flight can proceed using just pilotage, or a combination of pilotage and dead reckoning.

To the extent that a flight simulator is not entirely realistic, it must be due to one of two things. First, there can be hardware limitations. For example, the CPU and video refresh speeds of the computer may be insufficient to update the out-of-the-window view fast enough for a smooth, highly detailed animation, thereby producing both a choppy appearance and a detectable lag in processing the control inputs from the mouse. As we show below, these infidelities in the simulation can cause problems when landing the aircraft, because the degraded performance of the computer in conjunction with the beginner's natural tendency to overcontrol can easily result in a crash. The second reason a flight simulator may not represent some aspect of flight realistically is that the underlying theory used in the program is in some respect incomplete or incorrect - that is, it fails to accurately describe the real thing. In Microsoft FS 4.0, for example, the aircraft often stalls in an unrealistic fashion, with the nose pitching up rather than down. Stalls in FS 4.0 also fail to show the characteristic shudder associated with the stall of a real aircraft.'

\section{Skill Acquisition on Desktop Simulators}

We have employed flight simulators over the last 3 years to teach students a number of useful skills (Bradley, 1993; Bradley \& Abelson, 1994). At the most basic level, the task of simply flying the aircraft develops important perceptual-motor skills. Specifically, the student must master the intricate feedback relationship between his or her control inputs (by way of the stick, rudder, and throttle) and the resulting changes in the outside visual environment and the instruments. Understanding this feedback relationship allows the student to control the aircraft precisely, as in flaring the aircraft to land, performing aerobatic maneuvers, and staying on the glidepath when shooting visual or instrument approaches to the runway. Moreover, reading instruments to assess the state of the aircraft in a three-dimensional space requires information-processing skills. Flight in IFR conditionscalled blind flying - demands the highest level of skill in this area, because the pilot must be able to visualize the aircraft in relation to the outside world in the absence of external visual cues. Even the relatively simple task of flying under VFR conditions in the immediate vicinity (or traffic pattern) of the local airport requires spatialorientation skills. The student must develop a clear representation of the airport in relation to nearby landmarks and constantly monitor his or her position using this cognitive map. Cross-country flying is, of course, even more demanding and requires the student to master basic navigational and computational skills (e.g., how to read and use a compass, how to make time and distance calculations, how to correct for wind drift, etc.). Time and distance calculations require relatively simple algebraic operations $(t=d / v, d=v t)$, and drift correction requires trigonometry or at least an elementary understanding of right triangles. Also important for cross-country flying is fuel management (i.e., being able to calculate the fuel needed for a particular trip, and being able to verify that the rate of fuel consumption during the flight is as expected). ${ }^{2}$

An example of a fairly challenging project is described by Bradley (1993). He had students simulate Lindbergh's transatlantic flight of 1927. The 36-hour flight followed Lindbergh's great circle route from Long Island to Paris and employed an aircraft designed to be a close replication of the Spirit of St. Louis. This and similar projects suggest that desktop flight simulators can be useful for developing and sharpening a variety of skills. But just how realistic are they? That is, to what extent do such programs truly capture the important aspects of flying and navigating an aircraft? Before at- 
tempting to answer this question, we should briefly consider full-motion flight simulators, or what the FAA and many in the aviation community consider "real" flight simulators. ${ }^{3}$

\section{Full-Motion Flight Simulators}

In contrast to the desktop flight simulator, which runs on the typical home computer and costs $\$ 40-\$ 60$ (low end) or $\$ 300-\$ 900$ (high end), a full-motion flight simulator requires dedicated mini- or mainframe computers and costs between 10 and 20 million dollars. These simulators are so realistic that the FAA allows pilots to satisfy currency requirements by logging time on the simulator rather than flying the real thing. More significantly, an air transport pilot can obtain a rating in a new type of aircraft (say, a Boeing 767) solely by qualifying on an appropriate simulator (Forster, 1991). He or she can then fly as pilot-in-command in the actual aircraft with absolutely no previous experience in type except that provided-by the simulator.

Full-motion simulators have a cockpit mounted on a platform that can be moved up or down or banked left or right using a system of hydraulic actuators (see MacKay, 1994 , p. 20). A common misconception is that the orientation of the platform when in motion models the attitude of the aircraft with respect to the ground (e.g., if the platform is tilted up then the aircraft is climbing, and so on). In fact, the motions of the platform are used to induce accelerative, decelerative, and turning forces that arise when maneuvering the aircraft. These forces help the vestibular system to "feel" as if the aircraft is climbing, descending, or turning, and this enhances the realism of the simulation.

On the inside, a full-motion simulator is a more or less exact replication of the cock pit of the aircraft being simulated. Full wrap-around visuals are provided, and the instruments, switches, levers, and flight controls are accurately rendered. During flight, the changing forces on the controls (yoke and rudder) that result from variation in airspeed are faithfully replicated, ${ }^{4}$ as are the movements of the needles on the instruments. Auditory cues including wind and engine noise, radio chatter, and system alerts are also provided.

In preparation for this paper, the authors had the opportunity to fly full-motion simulators at American Airlines and the Naval Air Station at Brunswick, Maine. Without question, a 17 million dollar system does buy a total experience when it comes to flight simulation. However, one of the most significant differences between desktop and full-motion simulators-namely, that the latter provides motion cues to the vestibular system-turns out to be relatively less important than one might think. We had the opportunity to fly both simulators with the motion system turned off and were surprised to discover that when we initiated a climb, dive, or turn the aircraft really felt as if it were moving. The visual cues alone seemed sufficient to induce quite convincing sensations of motion. The technicians staffing the simulators confirmed that the sensation of move- ment produced by the visuals is so convincing that the motion system is really only needed for simulating turbulence and other abrupt motion transients.

Why do observers experience such powerful sensations of movement even when the motion system is deactivated? Since the observer is surrounded by wraparound visuals of a moving environment, the simulator cockpit probably gives rise to very potent induced motion effects (Duncker, 1929/1938; Wallach, 1959). In this case, the observer/pilot is the "target" and the view out-the-window is the "frame of reference." The larger moving frame induces motion in the smaller, stationary, target. Visually induced sensations of movement also have much in common with the phenomenon of visual capture. As shown by Rock and Victor (1964), when vision and touch are put in conflict - as in feeling a cube and looking at it through prisms that make it appear rectangular, or when running one's hand along the edge of a door while viewing it through curvature-inducing prisms - the observer reports that the object feels like it looks, even though the visual information is incorrect. Similarly, in the cock pit of a flight simulator (with motion deactivated), the nonveridical visual information depicting the movement of the aircraft through space dominates the veridical information coming from the vestibular system that indicates that the observer is, in fact, stationary.

\section{Desktop Flight Simulators}

In comparison with their full-motion counterparts, desktop flight simulators provide a more limited rendering of the flight experience. Motion cues are absent and auditory cues are confined to engine noise and, in some cases, transmissions from a simulated ATC or airport tower controller. The flight instruments are represented graphically, rather than in hardware as with full-motion simulators, and the out-the-window view is confined to the straight-ahead, although alternate views may be invoked by appropriate keystrokes. Control inputs are provided by moving a mouse, a joystick, a control yoke, or a similar input device. Movement of the control device fore and aft pitches the nose of the aircraft down and up, respectively, whereas movement to the left or right banks the aircraft in the corresponding direction. Many programs also support rudder pedals, which are needed to make coordinated turns, to sideslip the aircraft, and to enter and recover from spins. Since most users do not bother to install rudder pedals, flight simulator programs usually interlink the rudder and the control yoke so that coordinated turns are automatically insured.

Although defining and measuring the visual resolution of a desktop simulator running on a particular system can be quite complex (Kopke, 1994b), the quality of the visual detail provided by the most recent desktop flight simulators is surprisingly good. True, the resolution of desktop systems is nowhere near that provided by the full-motion simulators described above, but considering the overhead on the CPU required to support realtime animation, desktop flight simulators do a very cred- 
ible job of portraying the constantly changing external visual environment of the aircraft. For example, Microsoft Flight Simulator 5.0 depicts quite realistically both the instrument panel and the out-the-window view from the cockpit. One possible scenario in Microsoft F.S. 5.0 is the final approach to runway 36 at Meigs Field, a general aviation airport in Chicago. The city is on the left, and Lake Michigan is on the right. As the aircraft continues down the glidepath, the out-the-window view changes accordingly with the scene expanding radially outward from the projected touch-down spot. ${ }^{5}$ In contrast to a conventional animation, in which the contents of each frame are created and stored in advance, a flight simulator must create and display each frame as it goes, on the basis of the control inputs provided by the user. This virtual reality aspect of desktop flight simulators makes their performance all the more impressive.

Some desktop simulators are VFR only. They allow you to fly over a simulated landscape or scenery area, but they do not support flight in clouds or instrument conditions. Others are primarily IFR simulators. Although they have a small window to see out of, the only time you see anything is on arrival or departure from an airport because the enroute phase of the flight is always in clouds. The smaller out-the-window view allows the instrument panel of the aircraft to be depicted in far greater detail than in VFR simulators; for this reason, the IFR panel is more likely to include sophisticated navigational instrumentation, such as a radio magnetic indicator (RMI) or a horizontal situation indicator (HSI). Paradoxically, although flight in IFR conditions is more difficult than flight in VFR conditions, it is much more difficult to program a VFR simulator because the requirement to generate and display a constantly changing out-the-window view (i.e., to do real-time animation) taxes the computational capability of the computer to the limit. The mathematics required to compute and display the changing perspectives of objects seen from the aircraft are nontrivial, and programmers of VFR simulators devote considerable attention to finding the most efficient algorithms for doing this. In contrast, IFR simulators need only worry about updating the indications on the various instruments represented on the panel.

In addition to the VFR/IFR distinction, we can also distinguish low-end and high-end desktop flight simulators. A low-end simulator sells for under $\$ 100$ and is used as frequently for entertainment as it is for educational or training purposes. Low-end simulators tend to be VFR or a combination of VFR and IFR. High-end simulators sell for $\$ 300-\$ 900$ and are consistently IFR. The primary use of these simulators is for training and for maintaining proficiency. The developers of these programs hope that their products will eventually be recognized as a valid means for meeting at least some portion of the IFR training requirements (FAA, 1994). In addition to teaching IFR procedures, some high-end desktop simulators provide training in aircraft systems and in specific aircraft types.
Although the various desktop flight simulators on the market today differ in cost, sophistication, and the features offered, they have a number of limitations in common. We now consider these limitations and the impact that each has on the performance of computer pilots training on desktop systems.

\section{Limitations of Desktop Simulators}

Perhaps the first thing a licensed pilot notices when flying on a desktop simulator is the tunnel vision created by the limited dimensions of the computer monitor. Instead of a visual field extending $200^{\circ}$ from left to right (the horizontal span for unrestricted binocular vision), the view is limited to a meager $70^{\circ}$ or so. ${ }^{6}$ Also, because desktop simulators use the bottom portion of the monitor to display the instrument panel of the aircraft, the vertical visual field is also substantially truncated. To deal with these problems, desktop simulators allow you to display alternate views by pressing appropriate keys. For example, prior to turning from the downwind leg to the base leg of the traffic pattern, the pilot can select the view off the left wingtip in order to time the turn. However, the additional workload of pressing keys to go back and forth between views (as opposed to turning head and eyes), as well as the difficulty of flying the aircraft in one direction while looking in another, disrupts performance. Even experienced pilots find flying a precise pattern on a desktop simulator to be difficult, and overand undershooting are common when turning.

Another limitation of desktop simulators is that they provide only visual information about the state of the aircraft. Although auditory cues are sometimes available, most programs only simulate engine rpm. Changes in engine load and in the sound of the wind are not modeled, and these cues convey useful information when entering climbs, dives, turns, and stalls. The absence of vestibular cues was noted above: these cues help the pilot to "sense" the aircraft losing lift during an incipient stall, to detect the plane entering a bank (while preoccupied with an aeronautical chart), and so on. In fact, all of the G-forces produced by maneuvering the aircraft are missing, and this makes the computer pilot's task somewhat more difficult.

Another set of sensory cues missing from desktop flight simulators are those arising from the aerodynamic forces transmitted through the control system. At relatively high airspeeds the controls are stiff and difficult to move, while at low airspeeds the controls are "mushy" and require little effort to move. The effectiveness of a given control input also depends on airspeed: at high airspeeds, even small inputs can produce big effects; at low airspeeds, large control inputs are required to produce corresponding effects. Other examples of cues supplied through the controls are those associated with stalls. The pilot can usually sense a stall coming because the shudder produced by the turbulent flow of air over the wing is transmitted through the airframe and the control system. As the stall becomes more pronounced, the con- 
trols go slack and may flap or kick. Such cues are not currently modeled in desktop flight simulators, primarily because of the expense of designing control devices with the appropriate effectors to simulate control pressures and "induced" movements arising from stalls. However, some thought has been given to this matter (Moment, 1993), and we can anticipate that in the not too distant future desktop simulators will be available with "live" control systems.'

How detrimental are the various limitations mentioned above to the effectiveness of desktop flight simulators? The truncation of the visual field and the absence of nonvisual sensory cues make learning to fly on such simulators somewhat more difficult. As noted above, however, rather little advantage accrues to using the motion system available with full-motion simulators: most observers experience strong sensations of movement, induced through visual cues, even when the motion is deactivated. Although visually induced sensations of movement are not as powerful on desktop systems, this is probably due to the small visual angle of the image. When the output of a desktop flight simulator is projected to a large screen using a data projector, members of the audience experience strong sensations of motion during aerobatic demonstrations and routine flight maneuvers (Bradley \& Abelson, 1994). Of course, the best way to assess the effects of the limitations noted above is to conduct transfer studies. If substantial amounts of positive transfer occur from flying aircraft on desktop simulators to flying the real thing, then the impact of these limitations would seem to be minimal. ${ }^{8}$ There is one final limitation, however, that is nontrivial and that we have yet to discuss: the rate at which a desktop simulator displays the frames of the animation.

\section{The Frame Rate Problem}

We noted earlier that in order to produce the impression of an aircraft moving through space, the program has to create and display frames at a reasonable rate, say, 15-30 fps. Unfortunately, this is not always possible due to limitations in the processing speed of the computer system and the demands made on the simulation at a particular point in time (Kopke, 1994a). As the rates of movement and the number and complexity of the objects depicted in the out-the-window view go up, so do the computational demands on the program. At some point, there is simply so much to do in each cycle that the program is unable to construct the next frame in time to display it. This means that the current frame will stay up longer than it should, and the frame rate will drop below $15-30 \mathrm{fps}$. Furthermore, when the next frame finally is completed, the program has a choice to make. If it displays the late frame as soon as it is ready, then the continuity of the animation is preserved, although the display rate is decreased a bit. This has the effect of slowing things down, because events are unfolding more slowly on the screen (in real time) than they otherwise would. If the program is concerned with maintaining real-time accuracy - which is important for preserving time and distance relationships for navigation-then it must skip some frames in order to save time. Dropping frames allows the program to keep things happening on the screen in synchronization with real time. However, the animation now becomes very choppy, and noticeable stepping occurs in the objects depicted across successively displayed frames (Bradley \& Abelson, 1994). In programs having highly detailed scenery, such as Microsoft FS 5.0 , the frame rate may drop as low as $4-6 \mathrm{fps}$ during particularly computation-intensive periods. This means that over $75 \%$ of the information in the animation is being dropped out.

Dramatic "hits" on frame rate are most common when the computer pilot is on final approach to a runway surrounded by detailed scenery, because objects in the outthe-window view displace more quickly as the aircraft gets low to the ground. As the aircraft approaches the runway threshold, the frame rate drops and video chop becomes more and more noticeable. This is simply the result of the CPU getting overwhelmed by the processing demands of displaying numerous objects at high rates of relative displacement.

What are the consequences of frame rate degradation on pilot performance? Aside from the "jerky" appearance of the animation, one might think that the effects of video chop would be no more detrimental to performance than a truncated visual field or the absence of nonvisual cues and control force feedback. As it turns out, this is not the case. The popular literature on desktop flight simulators and the various on-line bulletin boards dedicated to this topic make frequent reference to the difficulty of landing in Microsoft Flight Simulator $(3.0,4.0,5.0)$. Even experienced pilots using the program for the first time find it extremely difficult to shoot good landings. This can hardly be due to lack of the requisite skill. So why is it so difficult to land aircraft with desktop simulators that provide richly detailed visual environments to fly in? An analysis of the pilot-plane feedback relationship provides one answer. In landing an aircraft, the pilot's task is to guide the aircraft down an invisible line, called the glidepath, which extends outward from the touch-down point on the runway. Deviations from the glidepath may occur in the horizontal or vertical dimensions, or both, and the pilot must immediately correct these by supplying appropriate inputs to the controls. As the aircraft rejoins the glidepath, the pilot must remove these corrections and resume the original approach configuration.

In order to make (and remove) the corrections required to keep the aircraft on the glidepath, the pilot must be able to observe the effects of the control inputs and adjust them as necessary. Now imagine introducing a delay between when a control input is supplied and when the resulting visual feedback of the effects of that input is displayed on the computer monitor. It seems reasonable to suppose that such a delay would cause the pilot to overcontrol the aircraft. The pilot expects the control input to produce a more or less immediate effect, and when it doesn't, the natural tendency is to supply addi- 
tional control input to get the aircraft moving in the desired direction. When the effects of the original control input are finally displayed, it is too late to remove the additional control input that was subsequently supplied, and the aircraft now moves in the desired direction but with far more correction than needed. In a series of escalating control actions, the pilot attempts to compensate for this by removing some of the original correction and, failing to see effects immediately, supplies more input than is required, and the whole process begins anew. The result is a pilot-induced oscillation (PIO) of increasing amplitude that can be stopped only by a patient and deliberate effort to suppress the tendency to overcontrol.

How does the preceding analysis apply to desktop flight simulators? In the approach to Meigs Field discussed earlier, the frame rate drops precipitously as the aircraft approaches the runway. Instead of staying on the screen for only $1 / 15^{-1 / 30}$ of a second, each frame may remain up for as long as $1 / 4-1 / 6$ of a second. This means that if the pilot supplies a control input to correct a deviation from the glidepath, the results of that correction will not be displayed immediately. Furthermore, since judging the effects of the correction requires judging rates of change over time, the fewer the frames that are displayed each second, the longer it will take the pilot to properly assess the effects of the correction. Consequently, the video chop produced by a limited frame rate introduces an artificial lag between the input of a control action and the visual display of the results of that action. This disruption of the temporal relationships of the pilot-plane control loop induces $\mathrm{PIO}$ and a corresponding deterioration in pilot performance on landing. 9

\section{Delayed Sensory Feedback}

The degradation in pilot performance associated with reduced frame rates has much in common with the phenomenon of delayed auditory feedback (Lee, 1950; Yates, 1963). In the typical experimental paradigm, the subject is asked to recite a passage by speaking into a microphone while simultaneously monitoring a recording of that speech played back through earphones. By use of a tape loop arrangement, a delay can be introduced between when a word is spoken and when it is heard in the earphones. Delayed auditory feedback turns out to be highly disruptive of normal speech: subjects stam- mer, repeat phrases, start and stop, alter the pitch and intensity of their vocalizations, and experience heightened stress while trying to read under such conditions. Maximum disruption is produced by a delay of $0.2 \mathrm{sec}$.

An even closer analogy to the frame rate problem is given by a delayed visual feedback paradigm. Smith (1962) had subjects trace patterns, such as the stars in Figure 1, and imposed either no delay or a $0.52-\mathrm{sec}$ delay between the subjects arm movements and the visual feedback displayed on a monitor. (Delayed feedback was achieved by a tape loop arrangement.) Figure 1 a shows a tracing with no delay, and Figure $1 \mathrm{~b}$ shows a tracing with the 0.52-sec delay (adapted from Smith, 1962, p. 83). The tracing in Figure $1 \mathrm{~b}$ illustrates that delayed visual feedback seriously degrades performance. The delay causes severe disturbances in motion organization, as well as strong emotional reactions in the subjects (primarily of frustration). Of special interest, given the discussion above, is the finding that tracing movements that require continuous visual guidance became very noticeably oscillatory (Smith, 1962, p. 83). We may characterize this as a subject-induced oscillation, or SIO. This finding reflects the same tendency to "overcontrol" in tracing the star as was noted above for pilots landing aircraft on desktop simulators. ${ }^{10}$

The frame rate problem differs in one important respect from the delayed visual feedback paradigm just described. The latter introduces a constant delay in the visual feedback, but the feedback is at all times continuous in that there are no "gaps" in the video recording as it plays on the monitor. When landing an aircraft under conditions of reduced frame rate, however, the visual feedback is intermittent, because some frames remain on the screen too long, and subsequent frames have to be skipped in order to maintain real-time synchronization. The question naturally arises as to whether or not the star-tracing task would show similar performance deficits when conducted under these conditions. To find out, Bradley and Abelson (1994) used a Panasonic video camera equipped with an electronic "strobe" feature. With the strobe activated, the camera samples the video signal every $1 / 6$ of a second, and each frame is displayed on the video monitor for $1 / 6$ of a second before the camera replaces it with another frame. The effect is precisely like that observed on the computer monitor when the
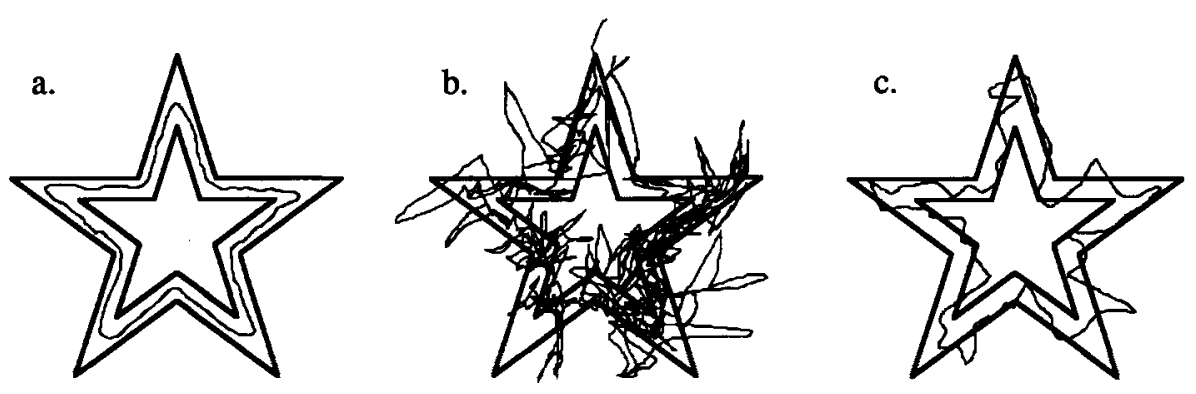

Figure 1. Star tracings under conditions of (a) no delay, (b) a 0.52 -sec delay with continuous visual feedback, and (c) intermittent visual feedback at 6 fps and frame durations of $0.17 \mathrm{sec}$. 
frame rate of a desktop simulator drops to 6 fps or so. To see the effects of delayed/intermittent feedback on the star-tracing task, Bradley and Abelson (1994) had a subject trace the star while viewing it and his arm on a monitor. The video signal sent to the monitor was supplied by the Panasonic camera with the strobe feature activated. The result is shown in Figure $1 \mathrm{c}$. It is apparent that delayed/intermittent feedback disrupts the tracing performance. The fact that the tracing is more accurate than the one in Figure $1 \mathrm{~b}$ is probably due to the difference in feedback delays $(0.17 \mathrm{vs} .0 .52 \mathrm{sec})$ rather than the nature of the feedback (intermittent vs. continuous).

\section{Conclusion}

The analysis offered here attributes the difficulty in landing aircraft with desktop simulators to the delayed (and intermittent) visual feedback produced by low frame rates. In summarizing his results, Smith (1962) makes the following observation:

No other experimental operation besides spatial displacement of vision creates such pervasive immediate disturbances in behavior as does delayed sensory feedback. Even a slight delay of a few hundredths of a second causes severe disruption or breakdown of normal movement integration, usually accompanied by some degree of emotional disturbance. (p. 90)

To overcome the problems of delayed sensory feedback, the processing speeds of desktop systems will have to improve enough that any delay is less than "a few hundredths of a second." Given the dramatic improvements in processing power in recent years, it is tempting to be optimistic on this score. However, this optimism is offset by the tendency of programmers to demand more than the current processor technology can support. For example, with the advent of the 486 processor, it finally became possible to land an aircraft in Microsoft 4.0 without significant PIO. Unfortunately, this performance gain was short-lived: as soon as the 486 became widely available, Microsoft released Version 5.0 of the flight simulator. Although the photorealistic imagery employed by this program is quite impressive, sustaining the imagery in a real-time animation overwhelms even a 486/66 processor. As a result, delayed sensory feedback is once again a serious problem. While Pentium and Power-PC-based machines promise to provide acceptable performance, flight simulation programmers will no doubt find creative ways to exhaust the surplus processing power of these chips, and the cycle will begin anew. In the future, programmers would be well advised to give more attention to the tradeoff between maintaining aircraft responsiveness and portraying a richly detailed visual environment.

\section{REFERENCES}

BradLEY, D. R. (1993, May). A simulation of Lindbergh's 1927 transatlantic flight using Microsoft Flight Simulator 4.0. Paper presented at MicroWings '93: The International Conference on Aviation Simulation, Cornell University, Ithaca, New York.
Bradley, D. R., \& Abelson, S. B. (1994, May). Flight simulation in the undergraduate curriculum. Paper presented at MicroWings ' 94 : The International Conference on Aerospace Simulation, Dallas. DunCKER, K. (1938). Induced motion. In W. D. Ellis (Ed.), A source book of Gestalt psychology (pp. 161-172). New York: Humanities Press. (Original work published 1929)

FAA (1994, May). Panel discussion with the FAA on flight sims. Paper presented at MicroWings '94: The International Conference on Aerospace Simulation, Dallas.

FORSTER, S. (1991). Technical requirements for a third-generation PCbased instrument flight simulator. In A. R. Sadelow (Ed.), $P C$ Based Instrument Flight Simulation: A first collection of papers (TS-Vol. 4, pp. 19-24). New York: American Society for Mechanical Engineers.

Gibson, J. J., Olum, P., \& Rosenblatt, F. (1955). Parallax and perspective during aircraft landings. American Journal of Psychology, 68, 372-385.

KOPKE, T. (1994a). The science of simulations: Frame rates exposed! MicroWings Magazine, 2(2), 39-40.

KOPKE, T. (1994b). The science of simulations: What is the resolution of my system? MicroWings Magazine, 2(1), 8-9.

LEE, B. S. (1950). Some effects of side-tone delay. Journal of the Acoustical Society of America, 22, 824-826.

MaCKAY, R. (1994). What's it like to fly in a real sim? MicroWings Magazine, 2(1), 20-24.

Mass, D. (1993). Graphics techniques for flight simulations. MicroWings Magazine, 1(3), 18-19.

Moment, S. (1993, May). Flight dynamics, control, and simulation. Paper presented at MicroWings '93: The International Conference on Aviation Simulation, Cornell University, Ithaca, New York.

PruYn, P. (1992, May). State-of-the-art computer graphics. Paper presented at CPAA Conference on Computer-Based Flight Simulation, Cornell University, Ithaca, New York.

Rock, I., \& VICTOR, J. (1964). Vision and touch: An experimentally created conflict between the senses. Science, 143, 594-596.

Sмiтн, K. U. (1962). Delayed sensory feedback and behavior. Philadelphia: Saunders.

WaLlach, H. (1959). The perception of motion. Scientific American, 201, 55-60.

WERTHEIMER, M. (1961). Experimental studies on the seeing of motion. In T. Shipley (Ed. and Trans.), Classics in psychology (pp. 10321088). New York: Philosophical Library. (Original work published 1912)

YATES, A. J. (1963). Delayed auditory feedback. Psychological Bulletin, 60, 213-232.

\section{NOTES}

1. In simple models of the flight dynamics of an aircraft, the programmer views the aircraft as a point-mass moving through space subject to the forces of gravity, lift, thrust, and drag. The linear displacements of the point-mass along three axes of motion characterize the long-term behavior of the aircraft in flight. This approach constitutes a 3-DOF (degrees-of-freedom) model that is relatively easy to implement on a PC. However, such a model does not incorporate the effects of inertial reactions about the three axes of motion, and these play an important role in determining the aircraft's stability in response to internal and external perturbations (Forster, 1991). To accurately model stability and control effects, the programmer must employ a 6-DOF model. Due to the improvement in processing speeds of the PC, 6-DOF models are now being implemented in high-end flight simulation programs, such as AzureSoft's ELITE.

2. Planning a cross-country trip also requires a number of problem-solving skills. The student must plot the overall course on an aeronautical chart and determine the true course and true heading (corrected for wind) as well as the magnetic course and magnetic heading (corrected for wind and magnetic variation) for each leg of the trip. A cruise power setting and altitude must be selected for the flight, and this will in turn determine the true airspeed (TAS) of the aircraft. On the basis of the airspeed, wind direction, and wind velocity, the ground speed for each leg of the trip is then calculated and used to estimate the total time enroute and the total amount of fuel required for the flight. 
If sufficient fuel reserves are not available for a nonstop trip, then the student must plan to land and refuel at one or more airports enroute.

3. The distinction made here between desktop simulators and fullmotion simulators evolved informally over the years in discussions by members of Compuserve and MicroWINGS. The intent is to distinguish PC-based computer programs from the "real thing," where the latter implicitly refers to a motion-based flight trainer. However, this distinction is neither industry standard nor entirely correct. The FAA specifies the technical requirements for "flight trainers" or simulators: these include motion, dynamic flight control loading, high-fidelity visual systems, and faithful recreations of the cockpit and flight characteristics of specific aircraft (Forster, 1991, p. 19). Technical requirements for "training devices" that do not employ motion, control loading, or visual simulation have also been developed, and such devices are approved for limited use in pilot training. To date, however, the FAA has not attempted to specify the minimum requirements for a PC-based training system or to indicate what role, if any, such systems might play in pilot training. Since some PC-based systems are more sophisticated than training devices currently in use, this issue is in need of review.

4. The simulation of control forces in a flight trainer is referred to as dynamic flight control loading.

5. This changing pattern of stimulation is called a flow pattern or expansion pattern and is an example of motion perspective. An expansion pattern provides good information about the aircraft's rate and angle of approach to the runway. The potential usefulness of this information to pilots was noted many years ago by Gibson, Olum, and Rosenblatt (1955).

6. Most readers are familiar with the strong effects of wrap-around visuals provided by special projecting facilities, such as those encountered at science museums. These effects are referred to as vection. Unfortunately, the effective field of view (FOV) of most desktop systems is simply too small to support vection (Kopke, 1994, personal communication).

7. At least one program currently on the market, Instrument Pilot by Precision Training Software, Inc., does appear to model some aspects of control stick behavior. With this program, the amount of stick displacement required to produce a given effect (say, a $5^{\circ}$ bank or change in pitch) is related to airspeed, with larger displacements being required at lower airspeeds. In addition, the stick displacement required to maintain a given rate of descent is dependent on the current trim position and airspeed of the aircraft. For example, if the aircraft is trimmed for level flight at cruise ( $107 \mathrm{kts}$ ) and the power reduced to achieve a 1,000 fpm descent at $90 \mathrm{kts}$, substantial back pressure on the stick is required to maintain the descent (unless the aircraft is retrimmed). And if this same descent is made at $80 \mathrm{kts}$, even more back pressure is required. By taking into account the relationship between trim position, airspeed, angle of attack, and stick deflection, Instrument Pilot gives a realistic "feel" to the flight controls.
8. Preliminary data collected at Embry-Riddle Aeronautical University (FAA, 1994) indicate that positive transfer does occur from PCbased flight simulators to a Frasca instrument trainer. The Frasca is an FAA-approved training device consisting of a dedicated computer, a desktop unit that contains full size instruments, and a yoke that has control loading. The next step is to investigate the transfer of flight skills acquired on PC-based systems to actual flying. On the basis of teaching a total of six courses on flight simulation, we can offer some anecdotal evidence. After 5 weeks of training on flight simulators, one student planned and then executed a simulated flight from Lewiston to Bangor, Maine. He was then given the opportunity to fly a Piper Archer over the same route, accompanied by a licensed pilot who served as the pilot-in-command (PIC). The student handled the controls from shortly after takeoff to just before touchdown at Bangor without any assistance from the PIC. The student also did all of the navigation without assistance. In another case, a student successfully executed three landings on his first flight without help from the PIC. While the latter is something we neither expect nor advise, it nevertheless provides strong anecdotal evidence as to the potential for skill transfer from PC-based simulators to actual flying.

9. This is sometimes referred to as the cue-synchronization problem. Whenever feedback-produced stimulation from different parts of the system are not fully synchronized in real time, the performance of the human operator will degrade.

10. Smith (1962, pp. 16-19) notes that interest in the detrimental effects of delayed sensory feedback can be traced back to World War II. The velocity tracking system used to guide the 0.50 -caliber machine guns in the belly turret of the B-17 is a good example. In this system, there was a delay of $0.5-1.0 \mathrm{sec}$ between when the operator aimed the sight and when the turret rotated into the desired position. Novice gunners therefore tended to "spin" the turret wildly about as they attempted to track another aircraft. In the early 1960s, the most advanced technical application that Smith could envision that would produce delayed sensory feedback was the earth-controlled guidance of vehicles on a planetary or lunar surface (p. 94). Since the round-trip transmission time between the earth and moon is $2-3 \mathrm{sec}$, real-time control of a lunar rover from earth would be exceptionally difficult. In the 1990 s, the problem of delayed sensory feedback is best exemplified by the flight simulator programs described in this paper and, more generally, by any of the virtual reality systems currently under development that present the observer with a "virtual world" that is dependent on the observer's actions. The most important factor limiting the success of these systems is the length of the delay (introduced by processing limitations) between an action and its sensory consequences.

(Manuscript received November 18, 1994; accepted for publication December 13, 1994.) 